\title{
On the reliability of analytical models to predict solute transport in a fracture network
}

\author{
C. Cherubini ${ }^{1}$, C. I. Giasi ${ }^{2}$, and N. Pastore ${ }^{2}$ \\ ${ }^{1}$ HydrISE, Institut Polytechnique LaSalle Beauvais, 19 rue Pierre Waguet, 60026 Beauvais Cedex, France \\ ${ }^{2}$ Polytechnical University of Bari, Bari, Italy \\ Correspondence to: C. Cherubini (claudia.cherubini@lasalle-beauvais.fr)
}

Received: 8 November 2013 - Published in Hydrol. Earth Syst. Sci. Discuss.: 6 December 2013

Revised: 28 April 2014 - Accepted: 29 April 2014 - Published: 24 June 2014

\begin{abstract}
In hydrogeology, the application of reliable tracer transport model approaches is a key issue to derive the hydrodynamic properties of aquifers.

Laboratory- and field-scale tracer dispersion breakthrough curves (BTC) in fractured media are notorious for exhibiting early time arrivals and late time tailing that are not captured by the classical advection-dispersion equation (ADE). These "non-Fickian" features are proven to be better explained by a mobile-immobile (MIM) approach. In this conceptualization the fractured rock system is schematized as a continuous medium in which the liquid phase is separated into flowing and stagnant regions.

The present study compares the performances and reliabilities of the classical MIM and the explicit network model (ENM), taking expressly into account the network geometry for describing tracer transport behavior in a fractured sample at bench scale. Though ENM shows better fitting results than MIM, the latter remains still valid as it proves to describe the observed curves quite well.

The results show that the presence of nonlinear flow plays an important role in the behavior of solute transport. First, the distribution of solute according to different pathways is not constant, but it is related to the flow rate. Second, nonlinear flow influences advection in that it leads to a delay in solute transport respect to the linear flow assumption. However, nonlinear flow is not shown to be related with dispersion. The experimental results show that in the study case the geometrical dispersion dominates the Taylor dispersion. However, the interpretation with the ENM shows a weak transitional regime from geometrical dispersion to Taylor dispersion for high flow rates. Incorporating the description of the
\end{abstract}

flow paths in the analytical modeling has proven to better fit the curves and to give a more robust interpretation of the solute transport.

\section{Introduction}

In fractured rock formations, the rock mass hydraulic behavior is controlled by fractures. In such aquifers, open and wellconnected fractures constitute high permeability pathways and are orders of magnitude more permeable than the rock matrix (Bear and Berkowitz, 1987; Berkowitz, 2002; Bodin et al., 2003; Cherubini, 2008; Cherubini and Pastore, 2011; Geiger et al., 2010; Neuman, 2005).

In most studies examining hydrodynamic processes in fractured media, it is assumed that flow is described by Darcy's law, which expresses a linear relationship between pressure gradient and flow rate (Cherubini and Pastore, 2010). Darcy's law has been demonstrated to be valid at low flow regimes (Reynolds number $(R e)<1$ ). For $R e>1$ a nonlinear flow behavior is likely to occur.

But in real rock fractures, microscopic inertial phenomena can cause an extra macroscopic hydraulic loss (Klov, 2000) which deviates flow from the linear relationship among pressure drop and flow rate.

To experimentally investigate fluid flow regimes through deformable rock fractures, Zhang and Nemcik (2013) carried out flow tests through both mated and non-mated sandstone fractures in triaxial cell. For water flow through mated fractures, the experimental data confirmed the validity of linear Darcy's law at low velocity. For larger water flow through 
non-mated fractures, the relationship between pressure gradient and volumetric flow rate revealed that the Forchheimer equation offers a good description for this particular flow process. The obtained experimental data show that Izbash's law can also provide an excellent description for nonlinear flow. They concluded that further work was needed to study the dependency of the two coefficients on flow velocity.

In fracture networks, heterogeneity intervenes even in solute transport: due to the variable aperture and heterogeneities of the fracture surfaces the fluid flow will seek out preferential paths (Gylling et al., 1995) through which solutes are transported.

Generally, the geometry of fracture network is not well known, and the study of solute transport behavior is based on multiple domain theory according to which the fractured medium is separated into two distinct domains: high velocity zones such as the network of connected fractures (mobile domain) where solute transport occurs predominantly by advection, and lower velocity zones such as secondary pathway, stagnation zones (almost immobile domain), such as the rock matrix.

The presence of steep concentration gradients between fractures and the matrix causes local disequilibrium in solute concentration, which gives rise to dominantly diffusive exchange between fractures and the matrix. This explains the non-Fickian nature of transport, which is characterized by breakthrough curves (BTCs) with early first arrival and long tails.

Quantifying solute transport in fractured media has become a very challenging research topic in hydrogeology over the last three decades (Nowamooz et al., 2013; Cherubini et al., 2009).

Tracer tests are commonly conducted in such aquifers to estimate transport parameters such as effective porosity and dispersivity, to characterize subsurface heterogeneity, and to directly delineate flow paths. Transport parameters are estimated by fitting appropriate tracer transport models to the breakthrough data.

In this context, analytical models are frequently employed, especially for analyzing tests obtained under controlled conditions because they involve a small number of parameters and provide physical insights into solute transport processes (Liu et al., 2011).

The advection-dispersion equation (ADE) has been traditionally applied to model tracer transport in fractures. However, extensive evidence has shown that there exist two main features that cannot be explained by the ADE: the early first arrival and the long tail of the observed BTCs. (Neretnieks et al., 1982; Becker and Shapiro, 2000; Jiménez-Hornero et al., 2005; Bauget and Fourar, 2008).

Several other models have been used to fit the anomalous BTCs obtained in laboratory tracer tests carried out in single fractures. Among those, the MIM (Van Genuchten and Wierenga, 1976), has showed to provide better fits of BTCs (Gao et al., 2009; Schumer et al., 2003; Feehley et al., 2010).
In the well-controlled laboratory tracer tests carried out by Qian et al. (2011) a was MIM proven to fit both peak and tails of the observed BTCs better than the classical ADE model.

Another powerful method to describe non-Fickian transport in fractured media is the continuous time random walk (CTRW) approach (Berkowitz et al., 2006) which is based on the conceptual picture of tracer particles undergoing a series of transitions of length $s$ and time $t$.

Together with a master equation conserving solute mass, the random walk is developed into a transport equation in partial differential equation form. The CTRW has been successfully applied for describing non-Fickian transport in single fractures (Berkowitz et al., 2001; Jiménez-Hornero et al., 2005).

Bauget and Fourar (2008) investigated non-Fickian transport in a transparent replica of a real single fracture. They employed three different models including ADE, CTRW, and a stratified model to interpret the tracer experiments.

As expected, the solution derived from the ADE equation appears to be unable to model long-time tailing behavior. On the other hand, the CTRW and the stratified model were able to describe non-Fickian dispersion. The parameters defined by these models are correlated to the heterogeneities of the fracture.

Nowamooz et al. (2013) carried out experimental investigation and modeling analysis of tracer transport in transparent replicas of two Vosges sandstone natural fractures.

The obtained BTCs were then interpreted using a stratified medium model that incorporates a single parameter permeability distribution to account for fracture heterogeneity, together with a CTRW model, as well as the classical ADE model.

The results confirmed poorly fitting BTCs for ADE. In contrast, the stratified model provides generally satisfactory matches to the data (even though it cannot explain the longtime tailing adequately), while the CTRW model captures the full evolution of the long tailing displayed by the BTCs.

Qian et al. (2011) experimentally studied solute transport in a single fracture (SF) under non-Darcian flow conditions which was found to closely follow the Forchheimer equation.

They also investigated the influence of the velocity contrast between the fracture wall and the plane of symmetry on the dispersion process, which was called "boundary layer dispersion" by Koch and Brady (1985). They affirmed that this phenomenon had to be considered if the thickness of the boundary layer was greater than the roughness of the fracture. On the other hand, if the thickness of the boundary layer was smaller than the roughness of the fractures, the recirculation zones inside the roughness cavities rather than the boundary layer would be more relevant for the dispersion process, and thus the hold-up dispersion would become important. Since smooth parallel planes were used for constructing the SF in their experiment, the fracture roughness and the hold-up dispersion were negligible. 
Bodin et al. (2007) developed the SOLFRAC program, which performs fast simulations of solute transport in complex 2-D fracture networks using the time domain random walk (TDRW) approach (Delay and Bodin, 2001) that makes use of a pipe network approximation. The code accounts for advection and hydrodynamic dispersion in channels, matrix diffusion, diffusion into stagnant zones within the fracture planes, mass sharing at fracture intersections, and other mechanisms such as sorption reactions and radioactive decay. Comparisons between numerical results and analytical BTCs for synthetic test problems have proven the accuracy of the model.

Zafarani and Detwiler (2013) presented an alternate approach for efficiently simulating transport through fracture intersections. Rather than solving the two-dimensional Stokes equations, the model relies upon a simplified velocity distribution within the fracture intersection, assuming local parabolic velocity profiles within fractures entering and exiting the fracture intersection. Therefore, the solution of the two-dimensional Stokes equations is unnecessary, which greatly reduces the computational complexity. The use of a time-domain approach to route particles through the fracture intersection in a single step further reduces the number of required computations. The model accurately reproduces mixing ratios predicted by high-resolution benchmark simulations.

As most of previous investigations of flow and transport in fracture networks considered Darcian flow, the behavior of the solute transport in fracture networks under nonDarcian flow conditions has been therefore poorly investigated. In fracture networks, different pathways can be identified through which solute is generally distributed as a function of the energy spent by solute particles to cross the path. In this context, the presence of nonlinear flow could play an important role in the distribution of the solutes according to the different pathways. In fact, the energy spent to cross the path should be proportional to the resistance to flow associated to the single pathway, which in nonlinear flow regime is not constant but depends on the flow rate. This means that by changing the boundary conditions, the resistance to flow varies and as a consequence the distribution of solute in the main and secondary pathways also changes, giving rise to a different behavior of solute transport.

In previous studies by Cherubini et al. $(2012,2013 a)$ the presence of nonlinear flow and non-Fickian transport in a fractured rock formation was analyzed at bench scale in laboratory tests. The effects of nonlinearity in flow have been investigated by analyzing hydraulic tests on an artificially created fractured limestone block of parallelepiped $\left(0.6 \times 0.4 \times 0.8 \mathrm{~m}^{3}\right)$ shape.

The flow tests regarded the observation of the volumes of water passing through different paths across the fractured sample. In particular, the inlet flow rate and the hydraulic head difference between the inlet and outlet ports were measured. The experimental results show evidence of a non-
Darcy relationship between flow rate and hydraulic head differences that is best described by a polynomial expression. Transition from viscous dominant regime to inertial dominant regime was detected. The experiments have been compared with a 3-D numerical model in order to evaluate the linear and non-linear terms of Forchheimer equation for each path.

Moreover, a tortuosity factor was determined that is a measure of the deviation of each flow path from the parallel plate model. A power law has been detected between the Forchheimer terms and the tortuosity factor, which means that the latter influences flow dynamics.

The non-Fickian nature of transport was investigated by means of tracer tests that regard the measurement of BTCs for saline tracer pulse across a selected path varying the flow rate. The observed experimental BTCs of solute transport were proven to be better modeled by the 1-D analytical solution of MIM. The carried out experiments show that there exists a pronounced mobile-immobile zone interaction that cannot be neglected, and that leads to a non-equilibrium behavior of solute transport. The existence of a non-Darcian flow regime has showed to influence the velocity field in that it gives rise to a delay in solute migration with respect to the predicted value assuming linear flow. Furthermore, the presence of inertial effects has proved to enhance nonequilibrium behavior. Instead, the presence of a transitional flow regime seems not to exert influence on the behavior of dispersion.

Herein, in order to give a more physical interpretation of the flow and transport behavior, we build on the work by Cherubini et al. (2013a) by interpreting the obtained experimental results of flow and transport tests by means of the comparison of two conceptual models: the 1-D single rate MIM and the 2-D explicit network model (ENM). Unlike the former, the latter expressly takes the fracture network geometry into account.

When applied to fractured media, the MIM approach does not explicitly take the fracture network geometry into account, but it conceptualizes the shape of fractures as 1-D continuous media in which the liquid phase is separated into flowing and stagnant regions. The convective dispersive transport is restricted to the flowing region and the solute exchange is described as a first-order process.

Unlike MIM, the ENM may allow one to understand the physical meaning of flow and transport phenomena (i.e., the meaning of long-time behavior of BTCs that characterize fractured media) and permits one to obtain a more accurate estimation of flow and solute transport parameters. In this model the fractures are represented as 1-D pipe elements and they form a 2-D pipe network.

It is clear that ENM needs to address the problem of parameterization. In fact, the transport parameters of each individual fracture should be specified, and this leads to more uncertainty in the estimation. 
Our overarching objective is therefore to investigate the performances and the reliabilities of MIM and ENM approaches to describe conservative tracer transport in a fractured rock sample.

In this particular way the present paper focuses attention on the effects of nonlinear flow regime on different features that depict the conservative solute transport in a fracture network, such as mean travel time, dispersion, dual porosity behavior, and distribution of solute into different pathways.

\section{Theoretical background}

\subsection{Nonlinear flow}

In the literature different laws are reported that account for the nonlinear relationship between velocity and pressure gradient.

A cubic extension of Darcy's law that describes pressure loss versus flow rate for low flow rates is the weak inertia equation:

$-\frac{\mathrm{d} p}{\mathrm{~d} x}=\frac{\mu}{k} \cdot v+\frac{\gamma \rho^{2}}{\mu} \cdot v^{3}$,

where $p\left(\mathrm{ML}^{-1} \mathrm{~T}^{-2}\right)$ is the pressure, $k\left(\mathrm{~L}^{2}\right)$ is the permeability, $\mu\left(\mathrm{ML}^{-1} \mathrm{~T}^{-1}\right)$ is the viscosity, $\rho\left(\mathrm{ML}^{-3}\right)$ is the density, $v\left(\mathrm{~L} \mathrm{~T}^{-1}\right)$ is the velocity, and $\gamma(\mathrm{L})$ is called the weak inertia factor.

In case of higher Reynolds numbers $(R e \gg 1)$ the pressure losses pass from a weak inertial to a strong inertial regime, described by the Forchheimer equation (Forchheimer, 1901), given by

$-\frac{\mathrm{d} p}{\mathrm{~d} x}=\frac{\mu}{k} \cdot v+\rho \beta \cdot v^{2}$,

where $\beta\left(\mathrm{L}^{-1}\right)$ is called the inertial resistance coefficient or non-Darcy coefficient.

Forchheimer law can be written in terms of hydraulic head:

$-\frac{\mathrm{d} h}{\mathrm{~d} x}=a^{\prime} \cdot v+b^{\prime} \cdot v^{2}$,

where $a^{\prime}\left(\mathrm{TL}^{-1}\right)$ and $b^{\prime}\left(\mathrm{TL}^{-2}\right)$ are the linear and inertial coefficient respectively equal to

$a^{\prime}=\frac{\mu}{\rho g k} ; b^{\prime}=\frac{\beta}{g}$.

In the same way, the relationship between flow rate $Q$ $\left(\mathrm{L}^{3} \mathrm{~T}^{-1}\right)$ and hydraulic head gradient can be written as follows:

$-\frac{\mathrm{d} h}{\mathrm{~d} x}=a \cdot Q+b \cdot Q^{2}$,

where $a\left(\mathrm{TL}^{-3}\right)$ and $b\left(\mathrm{~T}^{2} \mathrm{~L}^{-6}\right)$ are related to $a^{\prime}$ and $b^{\prime}$ :

$a=\frac{a^{\prime}}{\omega_{\mathrm{eq}}} ; b=\frac{b^{\prime}}{\omega_{\mathrm{eq}}}$, where $\omega_{\mathrm{eq}}\left(\mathrm{L}^{2}\right)$ represents the equivalent cross sectional area of fracture.

\subsection{Mobile-immobile model}

The mathematical formulation of the MIM for non-reactive solute transport is usually given as follows:

$$
\begin{aligned}
\frac{\partial c_{\mathrm{m}}}{\partial t} & =D \frac{\partial^{2} c_{\mathrm{m}}}{\partial x^{2}}-v \frac{\partial c_{\mathrm{m}}}{\partial x}-\alpha\left(c_{\mathrm{m}}-c_{\mathrm{im}},\right) \\
\beta \frac{\partial c_{\mathrm{im}}}{\partial t} & =\alpha\left(c_{\mathrm{m}}-c_{\mathrm{im}}\right),
\end{aligned}
$$

where $t(\mathrm{~T})$ is the time, $x(\mathrm{~L})$ is the spatial coordinate along the direction of the flow, $c_{\mathrm{m}}$ and $c_{\mathrm{im}}\left(\mathrm{ML}^{-3}\right)$ are the crosssectional averaged solute concentrations respectively in the mobile and immobile domain, $v\left(\mathrm{~L} \mathrm{~T}^{-1}\right)$ is the average flow velocity, $D\left(\mathrm{~L}^{2} \mathrm{~T}^{-1}\right)$ is the dispersion coefficient, $\alpha\left(\mathrm{T}^{-1}\right)$ is the mass exchange coefficient, and $\beta[-]$ is the mobile water fraction. For a non-reactive solute $\beta$ is equivalent to the ratio between the immobile and mobile cross-sectional area (-).

The solution of system Eq. (7) describing 1-D non-reactive solute transport in an infinite domain for instantaneous pulse of solute injected at time zero at the origin is given by (Goltz and Roberts, 1986):

$c_{\mathrm{m}}(x, t)=e^{-\alpha t} c_{0}(x, t)+\alpha \int_{0}^{t} H(t, \tau) c_{0}(x, \tau) \mathrm{d} \tau$,

where $c_{0}$ represents the analytical solution for the classical advection-dispersion equation (Crank, 1956):

$c_{0}(x, t)=\frac{M_{0}}{\omega_{\mathrm{eq}} \sqrt{\pi D t}} e^{-\frac{(x-v t)^{2}}{4 D t}}$,

where $M_{0}(\mathrm{M})$ is the mass of the tracer injected instantaneously at time zero at the origin of the domain. The term $H(t, \tau)$ presents the following expression:

$H(t, \tau)=e^{-\frac{\alpha}{\beta}(t-\tau)-\alpha \tau} \frac{\tau I_{1}\left(\frac{2 \alpha}{\beta} \sqrt{\beta(t-\tau) \tau}\right)}{\sqrt{\beta(t-\tau) \tau}}$,

where $I_{1}$ represents the modified Bessel function of the first kind .

In order to fit the BTCs with the MIM the assumption of representative 1-D length $(L)$ of the fracture network should be made. However, this matter can be solved by the introduction of the normalized velocity $(v / L)$ and normalized dispersion $\left(D / L^{2}\right)$. The MIM is defined by four parameters regarding the whole fracture network $\left(\nu / L, D / L^{2}, \alpha, \beta\right)$.

\subsection{Explicit network model}

Assuming that a SF $j$ can be represented by a 1-D pipe element, the relationship between head loss $\Delta h_{j}$ (L) and flow 
rate $Q_{j}\left(\mathrm{~L}^{3} \mathrm{~T}^{-1}\right)$ can be written in finite terms on the basis of Forchheimer model:

$$
\frac{\Delta h_{j}}{l_{j}}=a Q_{j}+b Q_{j}^{2} \Rightarrow \Delta h_{j}=\left[l_{j}\left(a+b Q_{j}\right)\right] Q_{j},
$$

where $l_{j}(\mathrm{~L})$ is the length of fracture, and $a\left(\mathrm{TL}^{-3}\right)$ and $b$ $\left(\mathrm{T}^{2} \mathrm{~L}^{-6}\right)$ are the Forchheimer parameters in finite terms.

The term in the square brackets represents the resistance to flow $R_{j}\left(Q_{j}\right)\left(\mathrm{TL}^{-3}\right)$ of $j$ fracture.

For a steady-state condition and for a 2-D simple geometry of the fracture network, the solution of flow field can be obtained in a straightforward manner applying the first and second Kirchhoff's laws.

The first law affirms that the algebraic sum of flow in a network meeting at a point is zero:

$\sum_{j=1}^{n} Q_{j}=0$

whereas the second law affirms that the algebraic sum of the head losses along a closed loop of the network is equal to zero:

$\sum_{j=1}^{n} \Delta h_{j}=0$.

Generally in a 2-D fracture network, the SF can be set in series and/or in parallel.

In particular, the total resistance to flow of a network in which the fractures are arranged in a chain is found by simply adding up the resistance values of the individual fractures.

In a parallel network, the flow breaks up by flowing through each parallel branch and recombining when the branches meet again. The total resistance to flow is found by adding up the reciprocals of the resistance values and then taking the reciprocal of the total. The flow rate crossing the generic fracture $j$ belonging to parallel circuits $Q_{j}$ can be obtained as follows:

$Q_{j}=\sum Q \frac{1}{R_{j}}\left(\sum_{i=1}^{n} \frac{1}{R_{i}}\right)^{-1}$,

where $\sum Q\left(\mathrm{~L} \mathrm{~T}^{-3}\right)$ is the sum of the discharge flow evaluated for the fracture intersection located at the inlet bond of $j$ fracture, whereas the term in brackets represents the probability of water distribution of $j$ fracture $P_{Q, j}$.

The BTCs at the outlet of the network $c_{\text {out }}(t)\left(\mathrm{ML}^{-3}\right)$, for an instantaneous injection, can be obtained as the summation of BTCs of each elementary path in the network. The latter can be expressed as the convolution product of the probability density functions of residence times in each individual fracture belonging to the elementary path. Using the convolution theorem, $c_{\text {out }}(t)$ can be expressed as follows:

$c_{\text {out }}(t)=\frac{M_{0}}{Q_{0}} F^{-1}\left[\sum_{i=1}^{N_{\mathrm{ep}}} \prod_{j=1}^{n_{f, i}} P_{\mathrm{c}, j} F\left(s_{j}\left(l_{j}, t\right)\right)\right]$, where $M_{0}(\mathrm{M})$ is the injected mass of solute, $F$ is the Fourier transform operator, $N_{\mathrm{ep}}$ is the number of elementary paths, $n_{f, i}$ is the number of fractures in $i$ elementary path, and $P_{\mathrm{c}, j}$ and $s_{j}\left(l_{j}, t\right)\left(\mathrm{T}^{-1}\right)$ represent the fraction of solute crossing the $\mathrm{SF}$ and the probability density function of residence time, respectively.

$P_{\mathrm{c}, j}$ can be estimated as the probability of the particle transition at the inlet bond of each individual SF. The rules for particle transition through fracture intersections play an important role in mass transport. In the literature, several models have been developed and tested in order to represent the mass transfer within fracture intersections. The simplest rule is represented by the "perfect mixing model" in which the mass sharing is proportional to the relative discharge flow rates.

The perfect mixing model assumes that the probability of particle transition of the fraction of solute crossing the SF can be written as follows:

$P_{\mathrm{c}, j}=\frac{Q_{j}}{\sum Q}$,

where $Q_{j}$ represents the flow rate in the single $j$ fracture. Note that $P_{Q, j}$ is equal to $P_{\mathrm{c}, j}$ if assuming the perfect mixing model valid.

It is clear that in order to know $s_{j}\left(l_{j}, t\right)$, the transport model and consequently the transport parameters of each SF need to be defined. Using the 1-D analytical solution of the advection-dispersion equation model (ADE) for pulse input $s_{j}\left(l_{j}, t\right)$ can be evaluated in a simple way:

$s_{j}\left(l_{j}, t\right)=\frac{Q_{j}}{\omega_{\mathrm{eq}, j} \sqrt{\pi D_{j} t}} e^{-\frac{\left(l_{j}-v_{j} t\right)^{2}}{4 D_{j} t}}$,

in which the velocity $v_{j}$ and dispersion $D_{j}$ relating to the generic $j$ fracture can be estimated through the following expression:

$$
\begin{aligned}
v_{j} & =\frac{Q_{j}}{\omega_{\mathrm{eq}, j}}, \\
D_{j} & =\alpha_{L, j} v_{j},
\end{aligned}
$$

where $\omega_{\mathrm{eq}, j}$ and $\alpha_{L, j}$ are the equivalent crossing area and the dispersion coefficient of $j$ fracture, respectively.

The ENM is defined by six parameters regarding each SF $\left(a, b, P_{Q}, \omega_{\mathrm{eq}}, \alpha_{L}\right.$, and $\left.P_{\mathrm{c}}\right)$.

\section{Material and methods}

\section{Flow and tracer tests}

The experimental setup has been already extensively discussed in Cherubini et al. (2013a), however, for the completeness in this section a summary is reported. The analysis of flow dynamics through the selected path (Fig. 1) regards the observation of water flow from the upstream tank 
to the flow cell with a circular cross section of 0.1963 and $1.28 \times 10^{-4} \mathrm{~m}^{2}$, respectively.

Initially at time $t_{0}$, the valves $a$ and $b$ are closed and the hydrostatic head in the flow cell is equal to $h_{0}$. The experiment begins with the opening of the valve $a$, which is reclosed when the hydraulic head in the flow cell is equal to $h_{1}$. Finally, the hydraulic head in the flow cell is reported to $h_{0}$ through the opening of the valve $b$. The experiment procedure is repeated, changing the hydraulic head of the upstream tank $h_{\mathrm{c}}$. The time $\Delta t=\left(t_{1}-t_{0}\right)$ required to fill the flow cell from $h_{0}$ to $h_{1}$ has been registered.

Given that the capacity of the upstream tank is much higher than that of the flow cell, it is reasonable to assume that during the experiments the level of the upstream tank $\left(h_{\mathrm{c}}\right)$ remains constant. Under this hypothesis the flow inside the system is governed by the equation:

$S_{1} \frac{\mathrm{d} h}{\mathrm{~d} t}=\Gamma(\Delta h)\left(h_{\mathrm{c}}-h\right)$,

where $S_{1}\left(\mathrm{~L}^{2}\right)$ and $h(\mathrm{~L})$ are respectively the section area and the hydraulic head of the flow cell; $h_{\mathrm{c}}(\mathrm{L})$ is the hydraulic head of upstream tank and $\Gamma(\Delta h)$ represents the hydraulic conductance term representative of both the hydraulic circuit and the selected path.

The average flow rate $\bar{Q}$ can be estimated by means of the volumetric method:

$\bar{Q}=\frac{S_{1}}{t_{1}-t_{0}}\left(h_{1}-h_{0}\right)$,

whereas the average hydraulic head difference $\overline{\Delta h}$ is given by

$\overline{\Delta h}=h_{\mathrm{c}}-\frac{h_{0}+h_{1}}{2}$.

In correspondence of the average flow rate and head difference is it possible to evaluate the average hydraulic conductance as follows:

$\bar{\Gamma}(\Delta h)=\frac{S_{1}}{t_{1}-t_{0}} \ln \left(\frac{h_{0}-h_{\mathrm{c}}}{h_{1}-h_{\mathrm{c}}}\right)$.

The inverse of $\bar{\Gamma}(\Delta h)$ represents the average resistance to flow $\bar{R}(\bar{Q})$.

The study of solute transport dynamics through the selected path has been carried out by means of a tracer test using sodium chloride. Initially a hydraulic head difference between the upstream tank and downstream tank is imposed. At $t=0$ the valve $a$ is closed and the hydrostatic head inside the block is equal to the downstream tank. At $t=10 \mathrm{~s}$ the valve $a$ is opened while at time $t=60 \mathrm{~s}$ a mass of solute equal to $5 \times 10^{-4} \mathrm{~kg}$ is injected into the inlet port through a syringe. The source release time (1 s) is very small, and therefore the instantaneous source assumption can be considered valid.

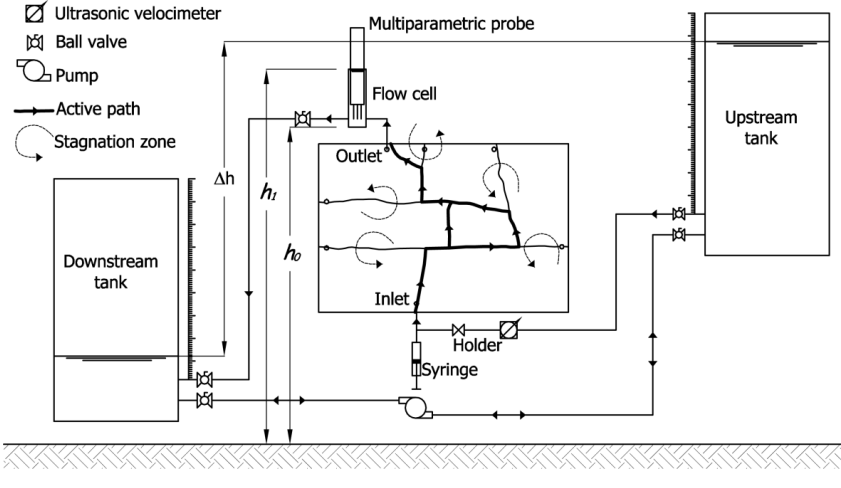

Figure 1. Schematic diagram of experimental setup.

In correspondence of the flow cell in which the multiparametric probe is located it is possible to measure the tracer BTC and the hydraulic head; in the meantime the flow rate entering the system is measured by means of an ultrasonic velocimeter. For different flow rates a BTC can be recorded at the outlet port.

Time moment analysis has been applied in order to characterize the BTCs in terms of mean breakthrough time, degree of spread, and asymmetry.

The mean residence time $t_{\mathrm{m}}$ is given by

$t_{\mathrm{m}}=\frac{\int_{0}^{\infty} t^{n} c(t) \mathrm{d} t}{\int_{0}^{\infty} c(t) \mathrm{d} t}$.

The $n$th normalized central moment of distribution of solute concentration versus time is defined as follows:

$\mu_{n}=\frac{\int_{0}^{\infty}\left[t-t_{\mathrm{m}}\right]^{n} c(t) \mathrm{d} t}{\int_{0}^{\infty} c(t) \mathrm{d} t}$.

The second moment $\mu_{2}$ represents the degree of spread relative to $t_{\mathrm{m}}$ whereas the degree of asymmetry measured by the skewness coefficient is defined as follows:

$S=\mu_{3} / \mu_{2}^{3 / 2}$.

\section{Discussion}

\subsection{Estimation of flow model parameters}

The flow field in each SF of the network can be solved in analytical way by means of Kirchhoff laws. In Fig. 2 the 2-D pipe network conceptualization is represented.

The resistance to flow of each single $j$ fracture is described by Eq. (12). The Forchheimer parameters are assumed constant for the whole fracture network. 
The application of the Kirchhoff's first law at the node 3 can be written as follows:

$Q_{0}-Q_{1}-Q_{2}=0$,

whereas the application of the Kirchhoff's second law at the loops $3-6$ can be written as follows:

$R_{6}\left(Q_{1}\right) Q_{1}-\left(R_{3}\left(Q_{2}\right)+R_{4}\left(Q_{2}\right)+R_{5}\left(Q_{2}\right)\right) Q_{2}=0$.

Substituting Eq. (27) into Eq. (28) the iterative equation of flow rate $Q_{1}$ can be obtained:

$$
\begin{aligned}
& Q_{1}^{k+1}=Q_{0} \\
& {\left[\frac{R_{3}\left(Q_{0}-Q_{1}^{k}\right)+R_{4}\left(Q_{0}-Q_{1}^{k}\right)+R_{5}\left(Q_{0}-Q_{1}^{k}\right)}{R_{3}\left(Q_{0}-Q_{1}^{k}\right)+R_{4}\left(Q_{0}-Q_{1}^{k}\right)+R_{5}\left(Q_{0}-Q_{1}^{k}\right)+R_{6}\left(Q_{1}^{k}\right)}\right] .}
\end{aligned}
$$

The Forchheimer parameters representative of whole fracture network can be derived matching the average resistance to flow derived experimentally with the resistance to flow evaluated for the whole network:

$$
\begin{aligned}
\bar{R}(\bar{Q})= & R_{1}\left(Q_{0}\right)+R_{2}\left(Q_{0}\right) \\
& +\left(\frac{1}{R_{6}\left(Q_{1}\right)}+\frac{1}{R_{3}\left(Q_{2}\right)+R_{4}\left(Q_{2}\right)+R_{5}\left(Q_{2}\right)}\right)^{-1} \\
& +R_{7}\left(Q_{0}\right)+R_{8}\left(Q_{0}\right)+R_{9}\left(Q_{0}\right) .
\end{aligned}
$$

Figure 3 shows the fitting of observed resistance to flow determined by the inverse of Eq. (23) and the theoretical resistance to flow (Eq. 30). The linear and nonlinear terms of Forchheimer model in Eq. (12) have been estimated and they are respectively equal to $a=7.345 \times 10^{4} \mathrm{~s} \mathrm{~m}^{-3}$ and $b=11.65 \times 10^{9} \mathrm{~s}^{2} \mathrm{~m}^{-6}$. It is evident that the 2-D pipe network model closely matches the experimental results $\left(r^{2}=\right.$ 0.9913). Flow characteristics can be studied through the analysis of Forchheimer number $F_{0}$ which represents the ratio of nonlinear to linear hydraulic gradient contribution:

$F o=\frac{b Q}{a}$.

Inertial forces dominate viscous ones at the critical Forchheimer number $(F O=1)$ corresponding in our case to a flow rate equal to $Q_{\text {crit }}=6.30 \times 10^{-6} \mathrm{~m}^{3} \mathrm{~s}^{-1}$, which is coherent with the results obtained in the previous study (Cherubini et al., 2013a).

The term in square brackets in Eq. (30) represents the probability of water distribution $P_{Q}$ evaluated for the branch 6. Note that it is not constant but it depends on the flow rate crossing the parallel branch. Figure 4 shows $P_{Q}$ as function of $Q_{0}$. The probability of water distribution decreases as the injection flow rate increases. This means that when the injection flow rate increases, the resistance to flow of the branch 6 increases faster than the resistance to flow of the branches $3-5$, and therefore the solute chooses the secondary pathway.

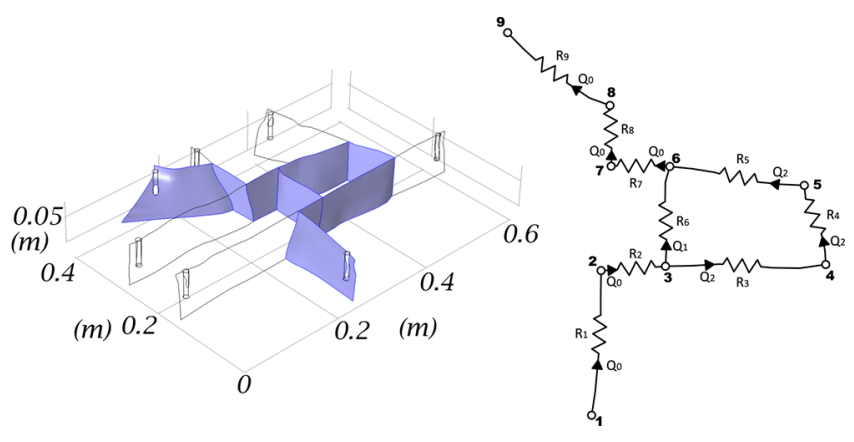

Figure 2. 2-D pipe network conceptualization of the fractured medium.

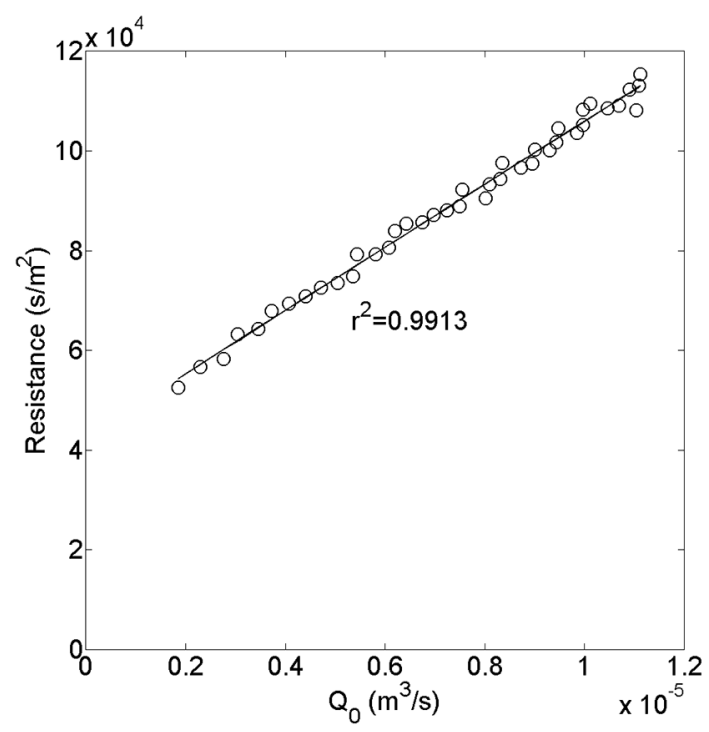

Figure 3. Average resistance to flow versus injection flow rate $\mathrm{Q}_{\mathbf{0}}$ $\left(\mathrm{m}^{3} \mathrm{~s}^{-1}\right)$. The circles represent the experimental values, the straight line represents the resistance to flow evaluated by Eq. (31).

\subsection{Fitting of breakthrough curves and interpretation of estimated transport model parameters}

Several tests have been conducted in order to observe solute transport behavior varying the injection flow rate in the range of $1.20 \times 10^{-6}-9.34 \times 10^{-6} \mathrm{~m}^{3} \mathrm{~s}^{-1}$. For each experimental BTC the mean travel time $t_{\mathrm{m}}$ and the coefficient of Skewness $S$ have been estimated.

Figure 5 shows $t_{\mathrm{m}}$ as function of $Q_{0}$. Travel time decreases more slowly for high flow rates. In particular, a change of slope is evident in correspondence of the injection flow rate equal to $4 \times 10^{-6} \mathrm{~m}^{3} \mathrm{~s}^{-1}$ (Cherubini et al., 2013a), which means the setting up of a transitional flow regime; the diagram of velocity profile is flattened because of inertial forces prevailing on the viscous one, as already shown by Cherubini et al. (2013a). The presence of a transitional flow regime leads to a delay on solute transport with respect to the values that can be obtained under the assumption of a linear flow field. Note that this behavior occurs before $Q_{\text {crit }}$. 


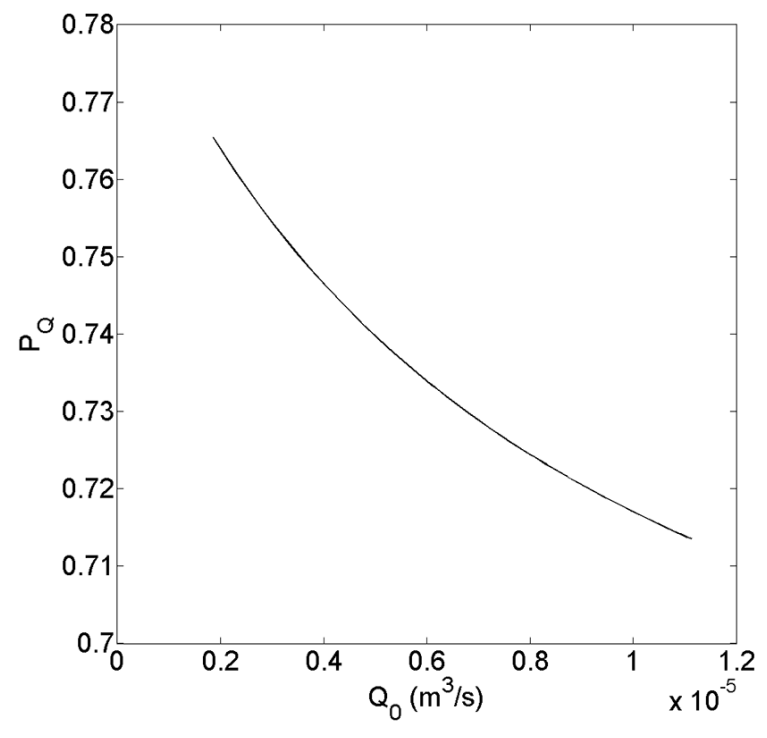

Figure 4. Probability of water distribution evaluated for main path $P_{Q}$ versus injection flow rate $Q_{0}\left(\mathrm{~m}^{3} \mathrm{~s}^{-1}\right)$.

The skewness coefficient does not exhibit a trend upon varying the injection flow rate, but its mean value is equal to 2.018. A positive value of skewness indicates that BTCs are asymmetric with early first arrival and long tail. This behavior seems not to be dependent on the presence of the transitional regime.

The measured BTCs for different flow rates have been individually fitted by MIM $\left(\nu / L, D / L^{2}, \alpha, \beta\right)$ and $\operatorname{ENM}\left(\omega_{\text {eq }}\right.$, $\left.\alpha_{\mathrm{L}}, P_{Q}, P_{\mathrm{C}}\right)$.

In particular, for the ENM the parameters $\omega_{\text {eq }}$ (equivalent area) and $\alpha_{L}$ are representative of all fracture network, whereas the parameters $P_{Q}$ and $P_{\mathrm{C}}$ are associated only to the parallel branches. For the considered fracture network Eq. (15) becomes

$c_{\text {out }}=\frac{M_{0}}{Q_{0}} F^{-1}\left[\begin{array}{l}P_{\mathrm{c}} \cdot F\left(s_{1}\right) \cdot F\left(s_{2}\right) \cdot F\left(s_{6}\right) \cdot F\left(s_{7}\right) \\ \cdot F\left(s_{8}\right) \cdot F\left(s_{9}\right)+\left(1-P_{\mathrm{c}}\right) \cdot F\left(s_{1}\right) \\ \cdot F\left(s_{2}\right) \cdot F\left(s_{3}\right) \cdot F\left(s_{4}\right) \cdot F\left(s_{5}\right) \\ \cdot F\left(s_{7}\right) \cdot F\left(s_{8}\right) \cdot F\left(s_{9}\right)\end{array}\right]$

The velocity and dispersion that characterize the probability density function $s$ are related to the flow rate that crosses each branch by Eqs. (18) and (19). This one is equal to the injection flow rate $Q_{0}$ except for branch 6 and branches 3-5 for which it is equal to $Q=P_{Q} Q_{0}$ and $Q=\left(1-P_{Q}\right) Q_{0}$, respectively.

Furthermore, three parameter configurations have been tested for the ENM. The configurations are distinguished on the basis of the number of fitting parameters and assumptions made on $P_{\mathrm{C}}$ and $P_{Q}$ parameters. The first configuration, named ENM2, has two fitting parameters $\omega_{\mathrm{eq}}$ and $\alpha_{L}$. In this configuration $P_{C}$ is imposed equal to $P_{Q}$ and is derived as the square brackets term in Eq. (29).

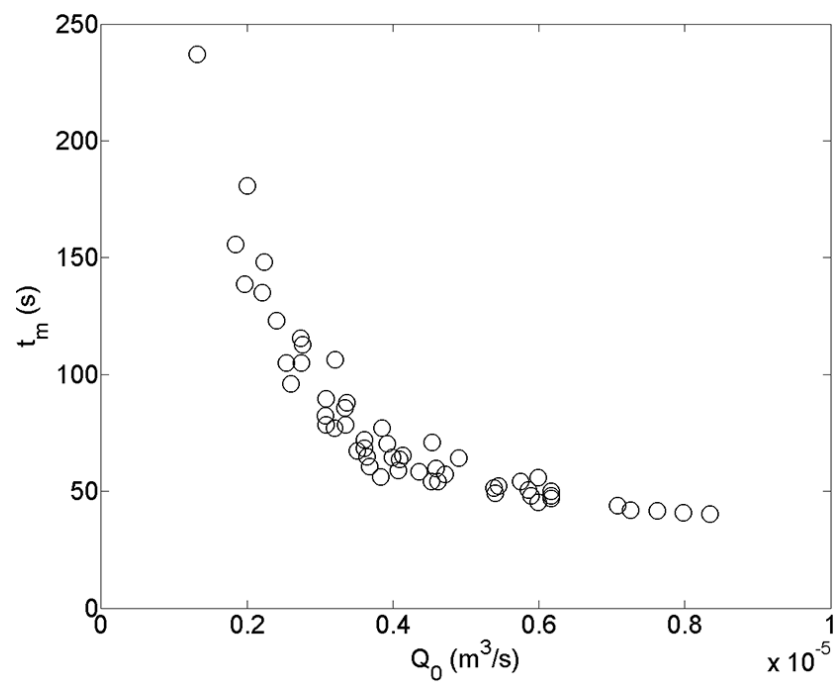

Figure 5. Mean travel time $t_{\mathrm{m}}$ (s) versus injection flow rate $Q_{0}$ $\left(\mathrm{m}^{3} \mathrm{~s}^{-1}\right)$.

The second configuration, named ENM3, has three fitting parameters $\omega_{\mathrm{eq}}, \alpha_{L}$, and $P_{C}\left(P_{Q}\right) . P_{\mathrm{C}}$ is still equal to $P_{Q}$ but they are evaluated by the interpretation of BTCs.

In the third configuration, named ENM4, all four parameters $\left(\omega_{\mathrm{eq}}, \alpha_{L}, P_{Q}, P_{C}\right)$ are determined through the fitting of BTCs.

To compare all the considered models, both the determination coefficient $\left(r^{2}\right)$ and the RMSE were used as criteria to determine the goodness of the fitting, which can be expressed as follows:

$r^{2}=1-\frac{\sum_{i=1}^{N}\left(C_{i, \mathrm{o}}-C_{i, \mathrm{e}}\right)^{2}}{\sum_{i=1}^{N}\left(C_{i, \mathrm{o}}-\bar{C}_{i, \mathrm{o}}\right)^{2}}$,

$\mathrm{RMSE}=\sqrt{\frac{1}{N} \sum_{i=1}^{N}\left(C_{i, \mathrm{o}}-C_{i, \mathrm{e}}\right)^{2}}$,

where $N$ is the number of observations, $C_{i, \mathrm{e}}$ is the estimated concentration, $C_{i, \mathrm{o}}$ is the observed concentration, and $\bar{C}_{i, \mathrm{o}}$ represents the mean value of $C_{i, \mathrm{o}}$.

Tables 1,2,3, and 4 show the estimated values of parameters, RMSE, and the determination coefficient $r^{2}$ for all the considered models varying the inlet flow rate $Q_{0}$.

Figure 6 shows the fitting results of BTCs for different injection flow rates.

For higher flow rates $\left(7.07 \times 10^{-6}\right.$ and $\left.4.80 \times 10^{-6} \mathrm{~m}^{3} \mathrm{~s}^{-1}\right)$ the fitting is poorer than for lower flow rates $\left(3.21 \times 10^{-6}\right.$ and $\left.1.96 \times 10^{-6} \mathrm{~m}^{3} \mathrm{~s}^{-1}\right)$. However, all models provide a satisfactory fitting. The ENM4 provides the highest values of $r^{2}$ varying in the range of $0.9921-1.000$ and the smallest values of RMSE in the range of 0.0033-0.0252. This is expected 
Table 1. Estimated values of parameters, RMSE, and determination coefficient $r^{2}$ for MIM at different injection flow rates in the fractured medium.

\begin{tabular}{cccccccc}
\hline $\begin{array}{c}\text { MIM } 1 \\
\text { No }\end{array}$ & $\begin{array}{c}Q \\
\left(\mathrm{~m}^{3} \mathrm{~s}^{-1}\right) \times 10^{-6}\end{array}$ & $\begin{array}{c}v / L \\
\left(\mathrm{~s}^{-1}\right) \times 10^{-2}\end{array}$ & $\begin{array}{c}D / L^{2} \\
\left(\mathrm{~s}^{-1}\right) \times 10^{-2}\end{array}$ & $\begin{array}{c}\alpha \\
\left(\mathrm{s}^{-1}\right) \times 10^{-2}\end{array}$ & $\begin{array}{c}\beta \\
(-)\end{array}$ & RMSE & $r^{2}$ \\
\hline 1 & 1.319 & $0.73 \pm 0.05$ & $0.15 \pm 0.01$ & $0.43 \pm 0.09$ & $0.95 \pm 0.14$ & 0.022 & 0.979 \\
5 & 2.209 & $1.05 \pm 0.05$ & $0.16 \pm 0.01$ & $0.50 \pm 0.12$ & $0.51 \pm 0.07$ & 0.021 & 0.991 \\
10 & 2.731 & $1.26 \pm 0.05$ & $0.18 \pm 0.01$ & $0.60 \pm 0.12$ & $0.51 \pm 0.06$ & 0.021 & 0.994 \\
15 & 3.084 & $1.74 \pm 0.06$ & $0.19 \pm 0.01$ & $1.03 \pm 0.16$ & $0.56 \pm 0.05$ & 0.023 & 0.995 \\
20 & 3.365 & $1.75 \pm 0.06$ & $0.20 \pm 0.01$ & $1.06 \pm 0.17$ & $0.54 \pm 0.05$ & 0.022 & 0.996 \\
25 & 3.681 & $2.49 \pm 0.10$ & $0.25 \pm 0.02$ & $1.67 \pm 0.32$ & $0.51 \pm 0.06$ & 0.030 & 0.995 \\
30 & 4.074 & $2.57 \pm 0.11$ & $0.26 \pm 0.02$ & $1.67 \pm 0.35$ & $0.50 \pm 0.06$ & 0.033 & 0.994 \\
35 & 4.536 & $2.25 \pm 0.09$ & $0.21 \pm 0.02$ & $1.58 \pm 0.29$ & $0.57 \pm 0.06$ & 0.031 & 0.994 \\
40 & 5.382 & $3.20 \pm 0.13$ & $0.26 \pm 0.02$ & $2.68 \pm 0.44$ & $0.61 \pm 0.06$ & 0.035 & 0.994 \\
45 & 5.895 & $3.32 \pm 0.15$ & $0.26 \pm 0.02$ & $2.82 \pm 0.50$ & $0.57 \pm 0.06$ & 0.036 & 0.995 \\
50 & 6.168 & $3.02 \pm 0.15$ & $0.26 \pm 0.02$ & $2.52 \pm 0.52$ & $0.51 \pm 0.07$ & 0.031 & 0.996 \\
55 & 8.345 & $3.54 \pm 0.29$ & $0.35 \pm 0.04$ & $3.05 \pm 1.07$ & $0.41 \pm 0.11$ & 0.038 & 0.995 \\
\hline
\end{tabular}

Table 2. Estimated values of parameters, RMSE, and determination coefficient $r^{2}$ for ENM2 at different injection flow rates in the fractured medium.

\begin{tabular}{cccccc}
\hline $\begin{array}{c}\text { ENM2 } \\
\text { No }\end{array}$ & $\begin{array}{c}Q \\
\left(\mathrm{~m}^{3} \mathrm{~s}^{-1}\right) \times 10^{-6}\end{array}$ & $\begin{array}{c}\omega_{\mathrm{eq}} \\
\left(\mathrm{m}^{2}\right) \times 10^{-4}\end{array}$ & $\begin{array}{c}\alpha_{L} \\
(\mathrm{~m}) \times 10^{-1}\end{array}$ & RMSE & $R^{2}$ \\
\hline 1 & 1.3194 & $3.10 \pm 0.14$ & $1.92 \pm 0.86$ & 0.033 & 0.952 \\
5 & 2.2090 & $3.22 \pm 0.04$ & $0.98 \pm 0.06$ & 0.020 & 0.993 \\
10 & 2.7312 & $3.29 \pm 0.04$ & $0.92 \pm 0.05$ & 0.019 & 0.995 \\
15 & 3.0842 & $2.81 \pm 0.03$ & $0.79 \pm 0.03$ & 0.020 & 0.996 \\
20 & 3.3648 & $3.06 \pm 0.03$ & $0.79 \pm 0.03$ & 0.019 & 0.997 \\
25 & 3.6813 & $2.35 \pm 0.02$ & $0.74 \pm 0.03$ & 0.026 & 0.996 \\
30 & 4.0735 & $2.49 \pm 0.02$ & $0.75 \pm 0.03$ & 0.027 & 0.996 \\
35 & 4.5356 & $3.27 \pm 0.04$ & $0.74 \pm 0.04$ & 0.028 & 0.995 \\
40 & 5.3824 & $2.76 \pm 0.02$ & $0.75 \pm 0.02$ & 0.023 & 0.998 \\
45 & 5.8945 & $2.90 \pm 0.02$ & $0.69 \pm 0.02$ & 0.027 & 0.997 \\
50 & 6.1684 & $3.30 \pm 0.04$ & $0.68 \pm 0.02$ & 0.032 & 0.995 \\
55 & 8.3455 & $3.56 \pm 0.05$ & $0.78 \pm 0.02$ & 0.041 & 0.994 \\
\hline
\end{tabular}

for two reasons. First, this model has more fitting parameters than ENM2 and ENM3, and thus it is more flexible. Second, compared to MIM it takes explicitly into account the presence of the secondary path.

The MIM considers the existence of immobile and mobile domains and a rate-limited mass transfer between these two domains. In the present context, this conceptualization can be a weak assumption, especially for high flow rates when the importance of secondary path increases. However, the fitting of BTCs shows that MIM remains valid as it proves to describe the observed curves quite well.

The extent of solute mixing can be assessed from the analysis of MIM first-order mass transfer coefficient $\alpha$ and the fraction of mobile water $\beta$.

Several authors have observed the variation of the masstransfer coefficient between mobile and immobile wa- ter regions with pore-water velocity (Van Genuchten and Wierenga, 1977; Nkedi-Kizza et al., 1984; De Smedt and Wierenga, 1984; De Smedt et al., 1986; Schulin et al., 1987). The increase in $\alpha$ with increasing water velocity is attributed to higher mixing in the mobile phase at high pore water velocities (De Smedt and Wierenga, 1984) or to shorter diffusion path lengths as a result of a decrease in the amount of immobile water (van Genuchten and Wierenga, 1977).

As concerns $\beta$, various authors have observed different behavior of the mobile water fraction parameter. Gaudet et al. (1977) reported increasing mobile water content with increasing pore water velocity. However, studies have also found that $\beta$ appears to be constant with varying pore-water velocity (Nkedi-Kizza et al., 1983). On the other hand, lower $\beta$ values can be attributed to faster initial movement of the solute as it travels through a decreasing number of faster flow paths. As a result, some authors have related $\beta$ values to the initial arrival of the solute. In fact, Gaudet et al. (1977) and Selim and Ma (1995) observed that the mobile water fraction parameter affects the time of initial appearance of the solute.

In general, the initial breakthrough time increases as $\beta$ increases (Gao et al., 2009), which can also be evidenced from Fig. 6. For lower flow rates the initial arrival time is higher than for higher flow rates. As the fraction of mobile water increases, the BTCs are shifted to longer times because the solute is transported through larger and larger fractions of the fracture volume. In the limiting case that the fraction of mobile water reaches 1, the MIM reduces to the equilibrium ADE (no immobile water) (Mulla and Strock, 2008).

The evidence of dual porosity behavior on solute transport is clearly shown by the analysis of the two MIM parameters: the ratio of mobile and immobile area $\beta$, and the mass exchange coefficient $\alpha$, shown in Fig. 7 as a function of velocity. 
Table 3. Estimated values of parameters, RMSE, and determination coefficient $r^{2}$ for ENM3 at different injection flow rates in the fractured medium.

\begin{tabular}{ccccccc}
\hline ENM 3 & \multicolumn{1}{c}{$\begin{array}{l}\alpha_{L} \\
\text { No. }\end{array}$} & $\begin{array}{c}Q \\
\left(\mathrm{~m}^{3} \mathrm{~s}^{-1}\right) \times 10^{-6}\end{array}$ & $\begin{array}{c}P_{Q} / P_{\mathrm{C}} \\
\left(\mathrm{m}^{2}\right) \times 10^{-4}\end{array}$ & $\begin{array}{c}\text { RMSE } \\
(\mathrm{m}) \times 10^{-1}\end{array}$ & $R^{2}$ \\
\hline 1 & 1.319 & $3.43 \pm 1.28$ & $1.92 \pm 0.86$ & $0.82 \pm 0.17$ & 0.032 & 0.954 \\
5 & 2.209 & $3.18 \pm 0.11$ & $0.98 \pm 0.06$ & $0.76 \pm 0.02$ & 0.020 & 0.993 \\
10 & 2.731 & $3.28 \pm 0.09$ & $0.92 \pm 0.05$ & $0.75 \pm 0.02$ & 0.019 & 0.995 \\
15 & 3.084 & $2.73 \pm 0.05$ & $0.79 \pm 0.03$ & $0.73 \pm 0.01$ & 0.019 & 0.997 \\
20 & 3.365 & $2.94 \pm 0.05$ & $0.79 \pm 0.03$ & $0.72 \pm 0.01$ & 0.017 & 0.997 \\
25 & 3.681 & $2.22 \pm 0.04$ & $0.74 \pm 0.03$ & $0.71 \pm 0.01$ & 0.023 & 0.997 \\
30 & 4.074 & $2.37 \pm 0.04$ & $0.75 \pm 0.03$ & $0.71 \pm 0.01$ & 0.025 & 0.997 \\
35 & 4.536 & $3.13 \pm 0.06$ & $0.74 \pm 0.04$ & $0.71 \pm 0.01$ & 0.026 & 0.995 \\
40 & 5.382 & $2.61 \pm 0.03$ & $0.75 \pm 0.02$ & $0.70 \pm 0.01$ & 0.016 & 0.999 \\
45 & 5.895 & $2.70 \pm 0.03$ & $0.69 \pm 0.02$ & $0.68 \pm 0.01$ & 0.016 & 0.999 \\
50 & 6.168 & $2.98 \pm 0.03$ & $0.68 \pm 0.02$ & $0.66 \pm 0.01$ & 0.017 & 0.999 \\
55 & 8.345 & $3.13 \pm 0.02$ & $0.78 \pm 0.02$ & $0.63 \pm 0.01$ & 0.016 & 0.999 \\
\hline
\end{tabular}

Table 4. Estimated values of parameters, RMSE, and determination coefficient $r^{2}$ for ENM4 at different injection flow rates in the fractured medium.

\begin{tabular}{|c|c|c|c|c|c|c|c|}
\hline \multicolumn{8}{|c|}{ ENM 4} \\
\hline No. & $\frac{Q}{\left(\mathrm{~m}^{3} \mathrm{~s}^{-1}\right) \times 10^{-6}}$ & $\begin{array}{c}\omega_{\mathrm{eq}} \\
\left(\mathrm{m}^{2}\right) \times 10^{-4}\end{array}$ & $\begin{array}{c}\alpha_{L} \\
(\mathrm{~m}) \times 10^{-1}\end{array}$ & $\begin{array}{l}P_{Q} \\
(-)\end{array}$ & $\begin{array}{l}P_{C} \\
(-)\end{array}$ & RMSE & $R^{2}$ \\
\hline 1 & 1.319 & $2.67 \pm 0.13$ & $1.18 \pm 0.11$ & $0.85 \pm 0.02$ & $0.67 \pm 0.02$ & 0.020 & 0.981 \\
\hline 5 & 2.209 & $3.15 \pm 0.12$ & $0.96 \pm 0.07$ & $0.76 \pm 0.02$ & $0.75 \pm 0.03$ & 0.020 & 0.993 \\
\hline 10 & 2.731 & $3.28 \pm 0.10$ & $0.92 \pm 0.06$ & $0.75 \pm 0.02$ & $0.76 \pm 0.02$ & 0.019 & 0.995 \\
\hline 15 & 3.084 & $2.74 \pm 0.06$ & $0.80 \pm 0.04$ & $0.73 \pm 0.01$ & $0.74 \pm 0.02$ & 0.019 & 0.997 \\
\hline 20 & 3.365 & $2.97 \pm 0.06$ & $0.81 \pm 0.04$ & $0.72 \pm 0.01$ & $0.73 \pm 0.02$ & 0.017 & 0.997 \\
\hline 25 & 3.681 & $2.28 \pm 0.05$ & $0.80 \pm 0.04$ & $0.70 \pm 0.01$ & $0.74 \pm 0.02$ & 0.020 & 0.998 \\
\hline 30 & 4.074 & $2.43 \pm 0.06$ & $0.80 \pm 0.04$ & $0.71 \pm 0.01$ & $0.74 \pm 0.02$ & 0.022 & 0.997 \\
\hline 35 & 4.536 & $3.18 \pm 0.08$ & $0.76 \pm 0.05$ & $0.71 \pm 0.01$ & $0.73 \pm 0.02$ & 0.025 & 0.996 \\
\hline 40 & 5.382 & $2.62 \pm 0.04$ & $0.76 \pm 0.03$ & $0.70 \pm 0.01$ & $0.70 \pm 0.01$ & 0.016 & 0.999 \\
\hline 45 & 5.895 & $2.76 \pm 0.03$ & $0.73 \pm 0.02$ & $0.68 \pm 0.01$ & $0.71 \pm 0.01$ & 0.014 & 0.999 \\
\hline 50 & 6.168 & $3.12 \pm 0.04$ & $0.76 \pm 0.02$ & $0.66 \pm 0.01$ & $0.71 \pm 0.01$ & 0.012 & 0.999 \\
\hline 55 & 8.345 & $3.46 \pm 0.02$ & $0.96 \pm 0.01$ & $0.63 \pm 0.00$ & $0.73 \pm 0.01$ & 0.003 & 1.000 \\
\hline
\end{tabular}

A different behavior of these two coefficients on varying the injection flow rate is observed in the present study. At Darcian-like flow conditions, the mass exchange coefficient remains constant, whereas the ratio of mobile and immobile area decreases as velocity increases. When nonlinear flow starts to become dominant, a different behavior is observed: $\alpha$ increases in a with a power function, whereas $\beta$ assumes a weakly growing trend as velocity increases with a mean value equal to 0.56 .

In order to better explain this behavior, the transport time (reciprocal of normalized velocity) and the exchange time (reciprocal of the exchange term) varying the flow rate for the MIM are shown in Fig. 8. In an analogous way the comparison between the mean travel time for the main path and the secondary path varying the injection flow rate for the ENM4 are shown in Fig. 4.

For the MIM at high flow rates, the exchange time joins the transport time; analogously for the ENM4, as the flow rate increases, the secondary path reaches the main path in terms of mean travel time. This analogous relationship between MIM and ENM enhances the concept that the mass transfer coefficient is dependent on flow velocity.

In Darcian-like flow conditions the main path is dominant over the secondary path. The latter can be considered as an immobile zone. In this condition the fracture network behaves as a SF, and the observed dual porosity behavior can be attributable only to the fracture-matrix interactions of the main path. 

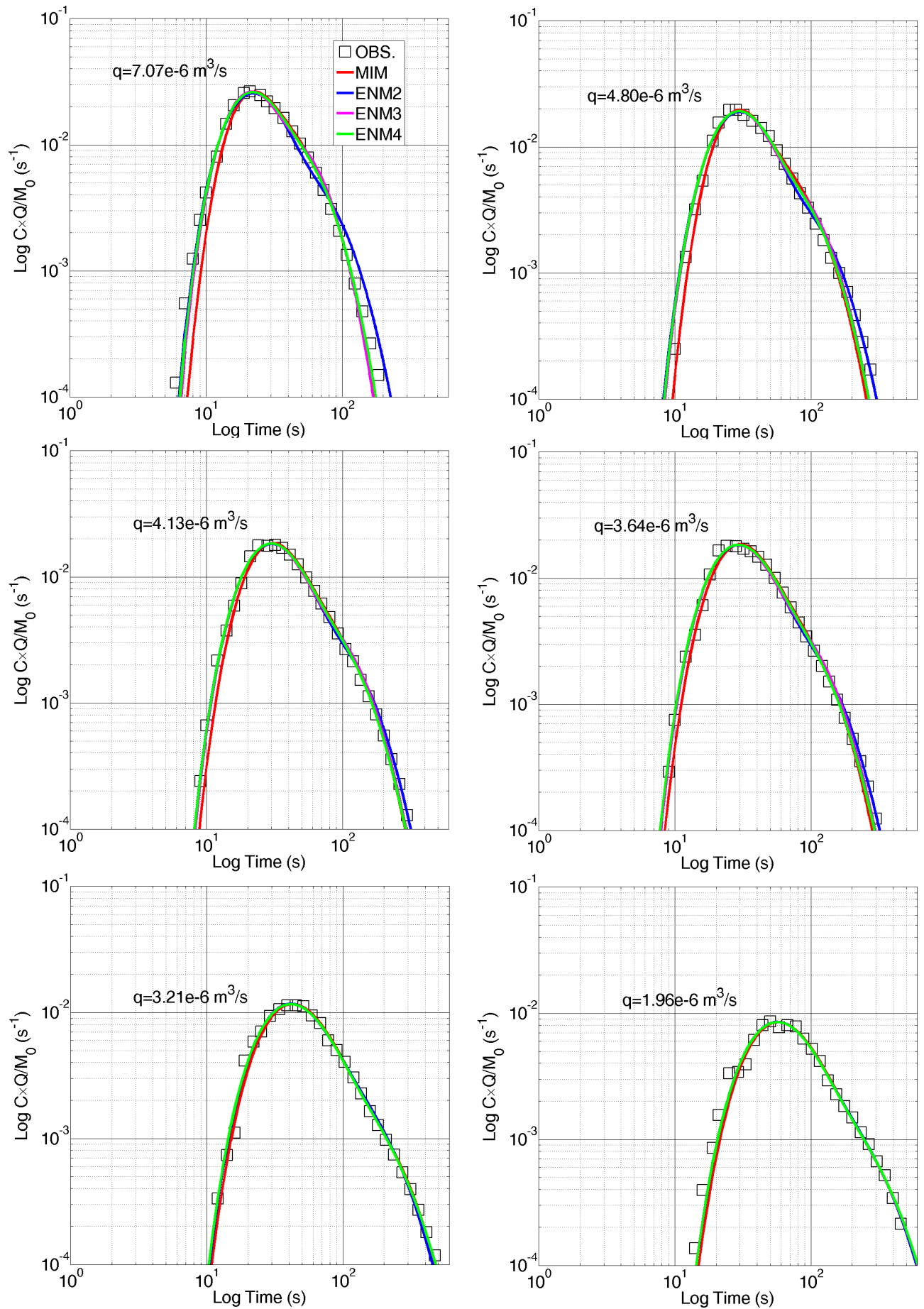

Figure 6. Fitting of BTCs at different injection flow rates using each of the four models (MIM, ENM1, ENM2, ENM3).

For higher velocities, a higher contact area between the mobile and immobile region is evidenced, enhancing solute mixing between these two regions (Gao et al., 2009). The increase in $\alpha$ with increasing water velocity is therefore attributable to nonlinear flow that enhances the exchange between the main and secondary flow paths. Increasing the injection flow rate, the importance of the secondary path grows and the latter cannot be considered as an immobile zone, as a consequence the dual porosity behavior becomes stronger.

As shown in Figs. 10 and $11, P_{Q}$ as a function of $Q_{0}$ evaluated by means of fitting the BTCs by ENM 3 and ENM4 presents a different trend in respect to $P_{Q}$ determined by means of flow tests. $P_{Q}$ evaluated by transport tests decreases more rapidly than $P_{Q}$ determined by flow tests (Fig. 10). In 

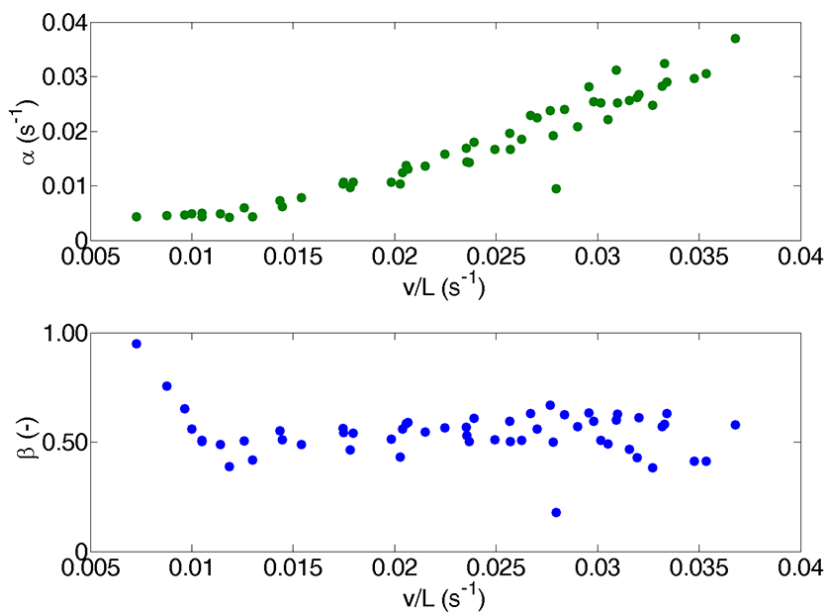

Figure 7. Mass transfer coefficient $\alpha$ and fraction of mobile water $\beta$ as function of normalized velocity $v / L\left(\mathrm{~s}^{-1}\right)$ for MIM. An outlier is evidenced for $v / L=0.028 \mathrm{~s}^{-1}$.

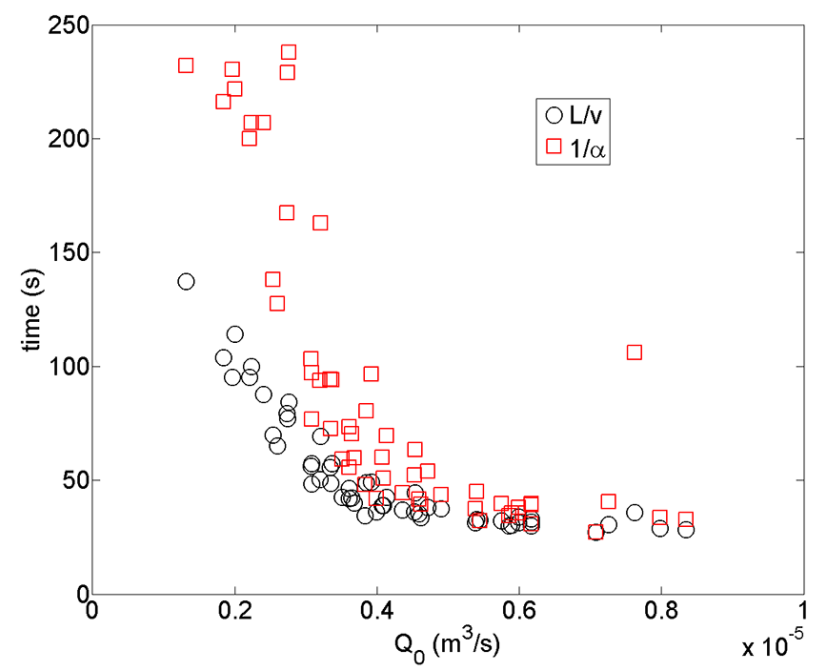

Figure 8. Transport time ( $L / v)$ (reciprocal of normalized velocity) and exchange time $(1 / \alpha)$ (reciprocal of the exchange term) as function of injection flow rate $Q_{0}\left(\mathrm{~m}^{3} \mathrm{~s}^{-1}\right)$ for MIM.

the ENM4, $P_{Q}$ and $P_{C}$ show a different behavior, and especially for higher velocity $P_{C}$ presents values higher than $P_{Q}$ (Fig. 11). In other words, the interpretation of BTCs evidences more enhanced nonlinear flow behavior than the flow tests.

In Fig. 12 the relationship between velocity $v$ and injection flow rate $Q_{0}$ is reported. Note that, in order to compare the results, the velocities for MIM are evaluated assuming the length of the medium equal to the length of main path ( $L=0.601 \mathrm{~m}$ ). Instead, for ENM4 the velocities are evaluated dividing $Q_{0}$ for the equivalent area $\omega_{\text {eq }}$. The models present the same behavior, and similar to the mean travel time a change of slope is evident again in correspondence of flow rate equal to $4 \times 10^{-6} \mathrm{~m}^{3} \mathrm{~s}^{-1}$. This result confirms

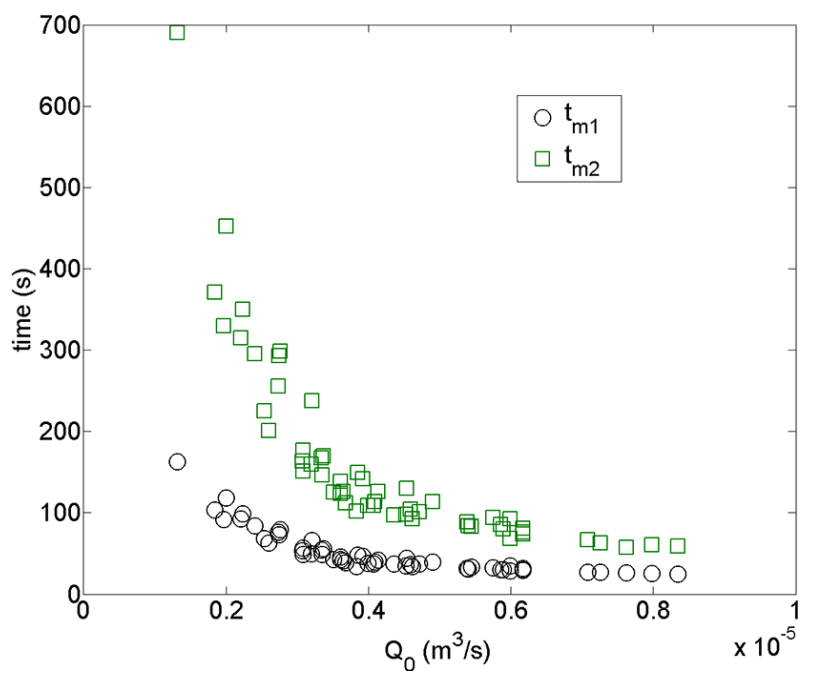

Figure 9. Travel time for main path $t_{\mathrm{m} 1}(\mathrm{~s})$ and travel time for secondary path $t_{\mathrm{m} 2}$ (s) for ENM4 as function of injection flow rate $Q_{0}$ $\left(\mathrm{m}^{3} \mathrm{~s}^{-1}\right)$.

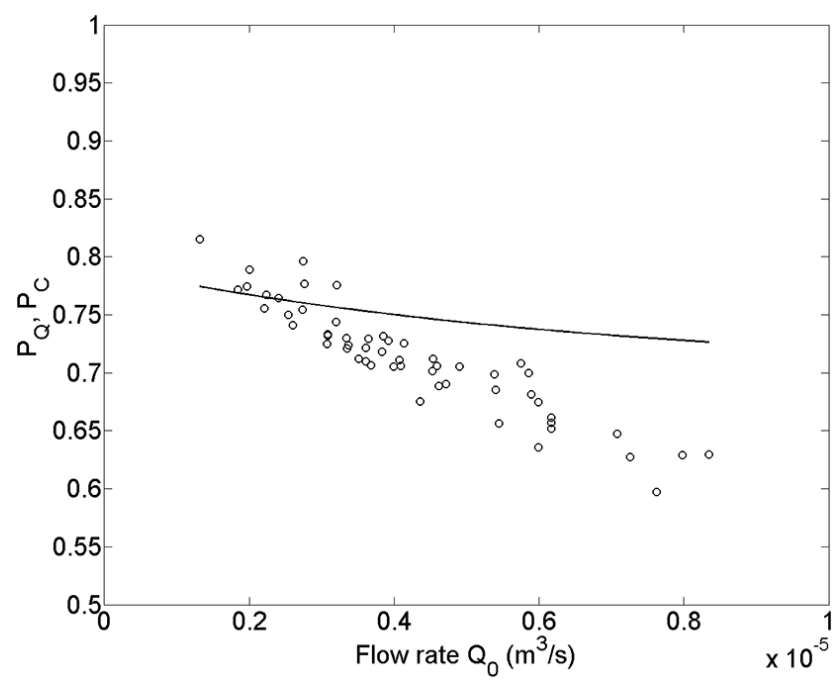

Figure 10. Comparison between the probability of water distribution $P_{Q}$ evaluated as the square brackets term in Eq. (29) (straight line) and the probability of particle transition $P_{C}\left(P_{Q}\right)$ for ENM3 (circle) varying the injection flow rate $Q_{0}\left(\mathrm{~m}^{3} \mathrm{~s}^{-1}\right)$.

the fact that the presence of nonlinear flow regime leads to a delay of solute transport with respect to the values that can be obtained under the assumption of a linear flow field.

In order to better represent the nonlinear flow regime, Fig. 13 shows water pressure as a function of velocity. A change of slope is evident for $v=1.5 \times 10^{-2} \mathrm{~m} \mathrm{~s}^{-1}$ which corresponds to the flow rate equal to $4 \times 10^{-6} \mathrm{~m}^{3} \mathrm{~s}^{-1}$.

Moreover, as shown in Fig. 14, a linear trend of dispersion with the injection flow rate both for MIM and ENM has been observed. This is coherent with what was obtained in the previous study (Cherubini et al., 2013a) where a linear 


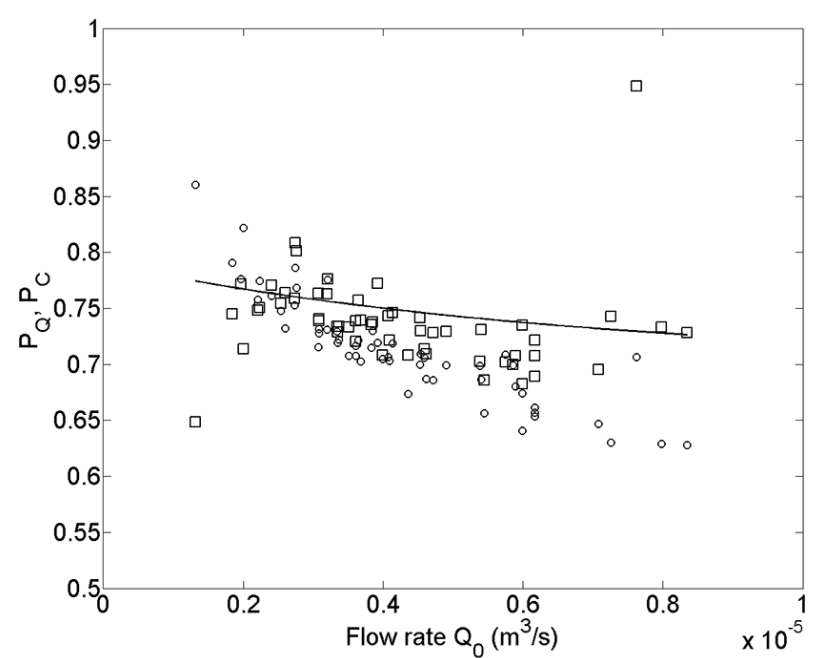

Figure 11. Comparison between the probability of water distribution $P_{Q}$ evaluated by the flow model (straight line) and the probability of particle transition $P_{\mathrm{c}}$ (square) and $P_{Q}$ (circle) for ENM4 varying the injection flow rate $Q_{0}\left(\mathrm{~m}^{3} \mathrm{~s}^{-1}\right)$.

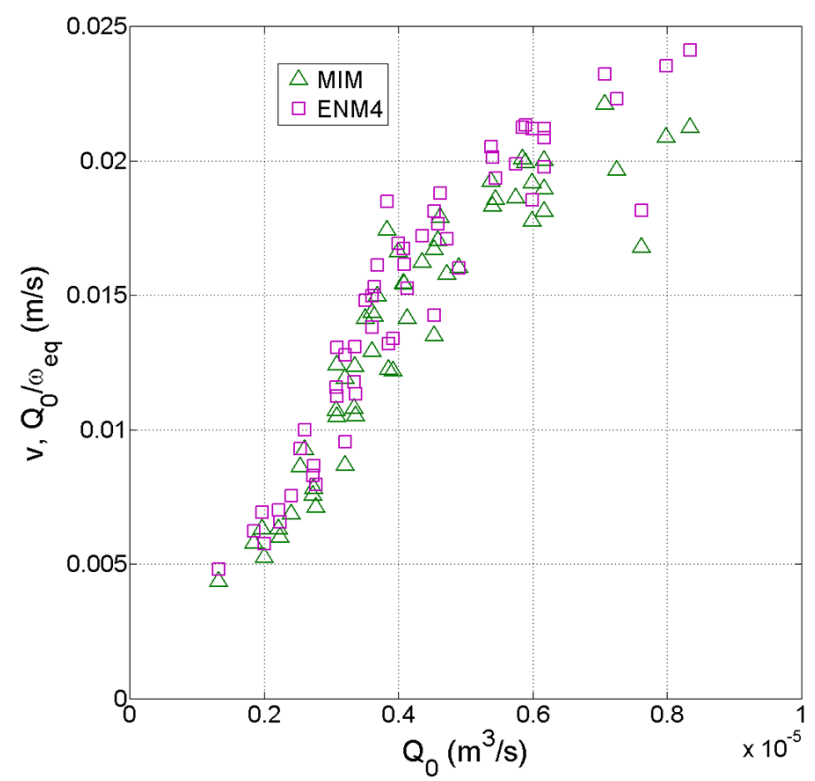

Figure 12. Velocity $v\left(\mathrm{~m} \mathrm{~s}^{-1}\right)$ as function of the injection flow rate $Q_{0}\left(\mathrm{~m}^{3} \mathrm{~s}^{-1}\right)$ for MIM and ENM4. Note that for MIM, the $v$ is determined assuming the length of medium equal to the length of main path $(L=0.601 \mathrm{~m})$. Instead for the ENM4, the velocity is determined dividing $Q_{0}$ for the equivalent area $\omega_{\mathrm{eq}}$.

relationship is found between velocity and dispersion both for ADE and MIM with the conclusion that geometrical dispersion dominated the effects of Aris-Taylor dispersion. The values of the coefficient of dispersion obtained for ENM do not depend on flow velocity but assume a somehow scattered but fluctuating value. Keeping $\alpha_{L}$ values constant, geometrical dispersion dominates the mixing processes along the

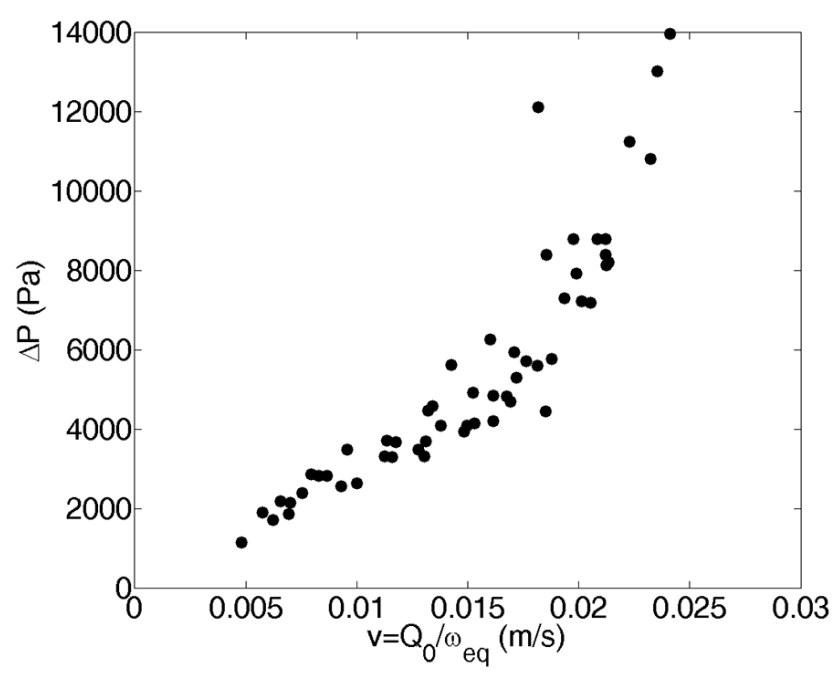

Figure 13. Difference of pressure $\Delta P(\mathrm{~Pa})$ as function of velocity $v$ $\left(\mathrm{m} \mathrm{s}^{-1}\right)$ for ENM4. The velocity is determined dividing $Q_{0}$ for the equivalent area $\omega_{\mathrm{eq}}$.

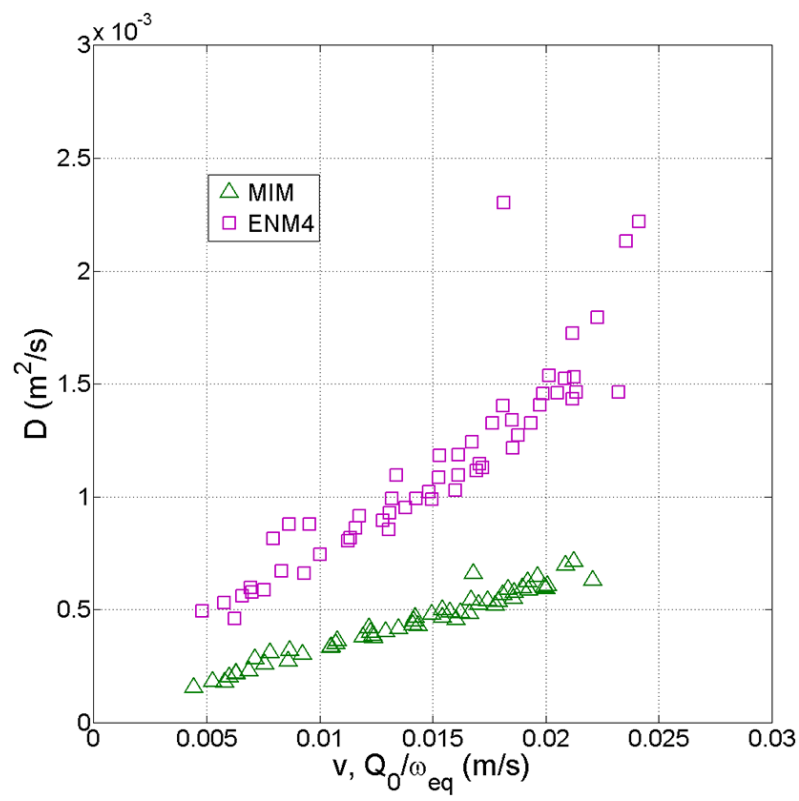

Figure 14. Dispersion $D\left(\mathrm{~m}^{2} \mathrm{~s}^{-1}\right)$ as function of velocity for MIM and ENM4. Note that for MIM, $D$ is determined assuming the length of the medium equal to the length of the main path $(l=0.601 \mathrm{~m})$. Instead for ENM4 $D$ is determined as $D=$ $Q_{0} \cdot \alpha_{L} / \omega_{\mathrm{eq}}$.

fracture network. Therefore, the presence of a nonlinear flow regime does not prove to exert any influence on dispersion except for high velocities for the ENM where a weak transitional regime appears.

This does not happen for MIM dispersion values whose rates of increase are smaller than those of ENM dispersion values. 
The values of the dispersion coefficient are in orders of magnitude of decimeters, which are comparable with the values obtained for Darcian condition (Qian et al., 2011), and the dispersion values of MIM are much lower than those of ENM.

This may be attributable to the fact that the MIM separates solute spreading into dispersion in the mobile region and mobile-immobile mass transfer. The dispersive effect is therefore partially taken into account by the mass transfer between the mobile zone and the immobile zone (Qian et al., 2011; Gao et al., 2009).

\section{Conclusions}

Flow and tracer test experiments have been carried out in a fracture network. The aim of the present study to compare the performances and reliabilities of two model paradigms: the mobile-immobile model (MIM) and the explicit network model (ENM) to describe conservative tracer transport in a fractured rock sample.

Fluid flow experiments show a non-negligible nonlinear behavior of flow best described by the Forchheimer law. The solution of the flow field for each SF highlights that the probabilities of water distribution between the main and the secondary path are not constant but decrease as the injection flow rate increases. In other words, with varying the injection flow rate the conductance of the main path decreases more rapidly than the conductance of the secondary path.

The BTCs determined by transport experiments have been fitted by MIM and three versions of ENM (ENM2, ENM3, ENM4) which differ on the basis of the assumptions made on the parameters $P_{Q}$ and $P_{\mathrm{C}}$. All models show a satisfactory fitting. The ENM4 provides the best fit, which is expected because it has more fitting parameters than ENM2 and ENM3, and thus it is more flexible. Additionally, compared to MIM, it takes explicitly into account the presence of the secondary path. Furthermore, for ENM the parameter $P_{Q}$ decreases more rapidly, varying the injection flow rate, than the same parameter determined by flow tests. The relationship between transport time and exchange time for MIM and mean travel time for main path and secondary path for the ENM4, varying the injection flow rate, has shown similarity of behavior: for higher values of flow rate the difference between transport time and exchange time decreases and the secondary path reaches the main path in terms of mean travel time. This relationship between MIM and ENM explains the fact that the mass transfer coefficient is dependent on flow velocity. The mass transfer coefficient increases as the importance of secondary path over the main path increases.

The velocity values evaluated for MIM and ENM show the same relationship with the injection flow rate. In particular, a change of slope is evident in correspondence of the flow rate equal to $4 \times 10^{-6} \mathrm{~m}^{3} \mathrm{~s}^{-1}$. This behavior occurs before the critical flow rate estimated by flow tests equal to $6.3 \times 10^{-6} \mathrm{~m}^{3} \mathrm{~s}^{-1}$. Therefore the interpretation of BTCs evidences more enhanced nonlinear behavior than flow tests. These results confirm the fact that the presence of transitional flow regime leads to a delay on solute transport with respect to the values that can be obtained under the assumption of a linear flow field (Cherubini et al., 2013a).

As concerns dispersion, a linear trend varying the velocity for both MIM and ENM has been observed - coherent with the previous results (Cherubini et al., 2013a) - the MIM underestimates the dispersion respect to ENM4.

The dispersivity values obtained for the different ENM models do not depend on flow velocity but assume a somehow scattered but fluctuating value. Keeping $\alpha_{L}$ values constant, geometrical dispersion dominates the mixing processes along the fracture network. Therefore, the presence of a nonlinear flow regime does not prove to exert any influence on dispersion except for high velocities for the ENM, where a weak transitional regime seems to appear. This result demonstrates that for our experiment, geometrical dispersion still dominates Taylor dispersion.

A major challenge for tracer tests modeling in fractured media is the appropriate selection of the modeling approach for each different study scale.

When dealing with large scales, tracer test BTCs are generally modeled by a relatively small number of model parameters (Becker and Shapiro, 2000).

At the laboratory scale, the definition of the network of fractures by means of discrete fracture network approaches (DFN) can permit to identify transport pathways and mass transport coefficients, in order to better define heterogeneous advective phenomena (Cherubini et al., 2013b).

At an intermediate local field scale $(1-100 \mathrm{~m})$, recognition that heterogeneous environments contain fast and slow paths led to the development of the MIM formulation applied successfully in a variety of hydrogeologic settings. However, the assumed velocity partitioning into flowing and notflowing zones is not an accurate representation of the true velocity field (Gao et al., 2009). Especially when the rock mass is sparsely fractured, the BTCs are characterized by early breakthrough and long tailing behavior, and a simple mobile-immobile conceptualization may be an over simplification of the physical transport phenomenon.

Solute transport in fractured aquifers characterized by highly non-Fickian behavior is therefore better described by an ENM rather than by a simple MIM. Applying a discrete model in such a case can permit to determine if transport occurs through one or several fractures and if multiple arrivals are caused by fracture heterogeneity, in such a way as to yield a more robust interpretation of the subsurface transport regime.

In such a context, geophysical imaging may provide detailed information about subsurface structure and dynamics (Dorn et al., 2012).

Edited by: M. Giudici 


\section{References}

Bauget, F. and Fourar, M.: Non-Fickian dispersion in a single fracture, J. Contam. Hydrol., 100, 137-148, doi:10.1016/j.jconhyd.2008.06.005, 2008.

Bear, J. and Berkowitz, B.: Groundwater flow and pollution in fractured rock aquifers, in: Developments in Hydraulic Engineering, edited by: Novak, P., Elsevier Applied Science Publishers Ltd., New York, 4, 175-238, 1987.

Becker, M. W. and Shapiro, A. M.: Tracer transport in fractured crystalline rock: evidence of nondiffusive breakthrough tailing, Water Resour. Res., 36, 1677-1686, doi:10.1029/2000WR900080, 2000.

Berkowitz, B.: Characterizing flow and transport in fractured geological media: a review, Adv. Water Resour., 25, 861-884, 2002.

Berkowitz, B., Kosakowski, G., Margolin, G., and Scher, H.: Application of continuous time random walk theory to tracer test measurements in fractured and heterogeneous porous media, Ground Water, 39, 593-604, 2001.

Berkowitz, B., Cortis, A., Dentz, M., and Scher, H.: Modeling non-Fickian transport in geological formations as a continuous time random walk, Rev. Geophys., 44, RG2003, doi:10.1029/2005RG000178, 2006.

Bodin, J., Delay, F., and de Marsily, G.: Solute transport in a single fracture with negligible matrix permeability: 1. fundamental mechanisms, Hydrogeol. J., 11, 418-433, 2003.

Bodin, J., Porel, G., Delay, F., Ubertosi, F., Bernard, S., and de Dreuzy, J.: Simulation and analysis of solute transport in 2D fracture/pipe networks: the SOLFRAC program, J. Contam. Hydrol., 89, 1-28, 2007.

Cherubini, C.: A modeling approach for the study of contamination in a fractured aquifer, in: Geotechnical and Geological Engineering, Springer, the Netherlands, 26, 519-533, 2008.

Cherubini, C. and Pastore, N.: Modeling contaminant propagation in a fractured and karstic aquifer, Fresen. Environ. Bull., 19, 1788-1794, 2010.

Cherubini, C. and Pastore, N.: Critical stress scenarios for a coastal aquifer in southeastern Italy, Nat. Hazards Earth Syst. Sci., 11, 1381-1393, doi:10.5194/nhess-11-1381-2011, 2011.

Cherubini, C., Giasi, C. I., and Pastore, N.: Application of Modelling for Optimal Localisation of Environmental Monitoring Sensors, Proceedings of the Advances in sensor and Interfaces (IWASI), Trani, Italy, 222-227, 2009.

Cherubini, C., Giasi, C. I., and Pastore, N.: Bench scale laboratory tests to analyze non-linear flow in fractured media, Hydrol. Earth Syst. Sci., 16, 2511-2522, doi:10.5194/hess-16-25112012, 2012.

Cherubini, C., Giasi, C. I., and Pastore, N.: Evidence of non-Darcy flow and non-Fickian transport in fractured media at laboratory scale, Hydrol. Earth Syst. Sci., 17, 2599-2611, doi:10.5194/hess17-2599-2013, 2013a.

Cherubini, C., Giasi, C. I., and Pastore, N.: Fluid flow modeling of a coastal fractured karstic aquifer by means of a lumped parameter approach, Environmental Earth Sciences, 70, 2055-2060, $2013 \mathrm{~b}$.

Crank, J.: Mathematics of Diffusion, Oxford University Press, New York, London, 1956

Delay, F. and Bodin, J.: Time domain random walk method to simulate transport by advection-dispersion and matrix diffusion in fracture networks, Geophys. Res. Lett., 28, 4051-4054, 2001.
De Smedt, F. and Wierenga, P. J.: Solute transfer through columns of glass beads, Water Resour. Res., 20, 225-232, 1984.

De Smedt, F., Wauters, F., and Sevilla, J.: Study of tracer movement through unsaturated sand, J. Hydrol., 85, 169-181, 1986.

Dorn, C., Linde, N., Le Borgne, T., Bour, O., and Klepikova, M.: Inferring transport characteristics in a fractured rock aquifer by combining single-hole ground-penetrating radar reflection monitoring and tracer test data, Water Resour. Res., 48, W11521, doi:10.1029/2011WR011739, 2012.

Feehley, C. E., Zheng, C., and Molz, F. J.: A dual-domain mass transfer approach for modeling solute transport in heterogeneous aquifers: Application to the Macrodispersion Experiment (MADE) site, Water Resour. Res., 36, 2501-2515, 2010.

Forchheimer, P.: Wasserbewegung durch Boden, Z. Verein Deut. Ing., 45, 1781-1788, 1901 (in German).

Gao, G., Zhan, H., Feng, S., Huang, G., and Mao, X.: Comparison of alternative models for simulating anomalous solute transport in a large heterogeneous soilcolumn, J. Hydrol., 377, 391-404, 2009.

Gaudet, J. P., Jégat, H., Vachaud, G., and Wierenga, P. J.: Solute transfer, with exchange between mobile and stagnant water, through unsaturated sand, Soil Sci. Soc. Am. J., 41, 665-671, 1977.

Geiger, S., Cortis, A., and Birkholzer, J. T.: Upscaling solute transport in naturally fractured porous media with the continuous time random walk method, Water Resour. Res., 46, W12530, doi:10.1029/2010WR009133, 2010.

Goltz, M. N. and Roberts, P. V.: Three-dimensional solutions for solute transport in an infinite medium with mobile and immobile zones, Water Resour. Res., 22, 1139-1148, 1986.

Gylling, B., Moreno, L., and Neretnieks, I.: Transport of solute in fractured media, based on a channel network model, in: Proceedings of Groundwater Quality: Remediation and Protection Conference, 14-19 May, edited by: Kovar, K. and Krasny, J., Prague, 107-113, 1995.

Jiménez-Hornero, F. J., Giráldez, J. V., Laguna, A., and Pachepsky, Y.: Continuous time randomwalks for analyzing the transport of a passive tracer in a single fissure, Water Resour. Res., 41, W04009, doi:10.1029/2004WR003852, 2005.

Klov, T.: High-velocity flow in fractures, Dissertation for the partial fulfillment of the requirements for the degree of doktor ingenieur Norvegian University of Science Technology Department of Petroleum Engineering and Applied Geophysics, Trondheim, 2000.

Koch, D. L. and Brady, J. F.: Dispersion in fixed beds, J. Fluid Mech., 154, 399-427, 1985.

Liu, H. H., Mukhopadhyay, S., Spycher, N., and Kennedy, B. M.: Analytical solutions of tracer transport in fractured rock associated with precipitation-dissolution reactions, Hydrogeol. J., 19, 1151-1160, 2011.

Mulla, D. J. and Strock, J. S.: Nitrogen transport processes in soil, in: Nitrogen in agricultural systems, edited by: Schepers, J. S. and Raun, W. R., Agron. Monogr. 49, ASA, CSSA, SSSA, Madison, WI, 401-436, 2008.

Neretnieks, I., Eriksen, T., and Tahtinen, P.: Tracer movement in a single fissure in granitic rock: some experimental results and their interpretation, Water Resour. Res., 18, 849-858, doi:10.1029/WR018i004p00849, 1982. 
Neuman, S. P.: Trends, prospects and challenges in quantifying flow and transport through fractured rocks, Hydrogeol. J., 13, 124147, 2005.

Nkedi-Kizza, P., Biggar, J. W., Selim, H. M., van Genuchten, M. Th., Wierenga, P. J., Davidson, J. M., and Nielson, D. R.: On the equivalence of two conceptual models for describing ino exchange during transport an aggregated oxisol, Water Resour. Res., 20, 1123-1130, 1984.

Nowamooz, A., Radilla, R., Fourar, M., and Berkowitz, B.: NonFickian Transport in Transparent Replicas of Rough-Walled Rock Fractures Transport in Porous Media July 2013, 98, 651682, doi:10.1007/s11242-013-0165-7, 2013.

Qian, J. Z., Chen, Z., Zhan, H. B., and Luo, S. H.: Solute transport in a filled single fracture under non-Darcian flow, Int. J. Rock Mech. Min., 48, 132-140, 2011.

Schulin, R., Wierenga, P. J., Fluhler, H., and Leuenberger, J.: Solute transport through a stony soil, Soil Sci. Soc. Am. J., 5, 36-42, 1987.
Schumer, R., Benson, D. A., Meerschaert, M. M., and Baeumer, B.: Fractal mobile/immobile solute transport, Water Resour. Res., 39, 1296, doi:10.1029/2003WR002141, 2003.

Selim, H. M. and Ma, L.: Transport of reactive solutes in soils: a modified two region approach, Soil Sci. Soc. Am. J., 59, 75-82, 1995.

Van Genuchten, M. T. and Wierenga, J.: Mass transfer studies in sorbing porous media, I - Analytical solutions, SSSA Proceedings, 40, 473-480, 1976.

Van Genuchten, M. T. and Wierenga, P. J.: Mass transfer studies in sorbing porous media: experimental evaluation with tritium, Soil Sci. Soc. Am. J., 41, 272-278, 1977.

Zafarani, A. and Detwiler, R. L.: An efficient time-domain approach for simulating Pe-dependent transport through fracture intersections, Adv. Water Resour., 53, 198-207, 2013.

Zhang, Z. and Nemcik, J.: Fluid flow regimes and nonlinear flow characteristics in deformable rock fractures, J. Hydrol., 477, 139-151, 2013. 